\section{Hepatitis colestásica probablemente inducida por ticlopidina en mujer con hipotiroidismo}

\section{Sr. Director:}

La ticlopidina es un antiagregante plaquetario que actúa inhibiendo la unión ADP-dependiente del fibrinógeno a la membrana plaquetaria sin inhibición de la ciclooxigenasa ${ }^{1}$. Este fármaco se emplea como prevención secundaria de los accidentes cerebrovasculares y en accidentes isquémicos transitorios en los casos en los que esté contraindicado el ácido salicílico o en los que haya fallado este fármaco ${ }^{2}$. A pesar de ser un fármaco con pocos efectos adversos, cuando se presentan son de una entidad tan llamativa que les hacen fácilmente reconocibles (neutropenia, trombopenia o hipercolesterolemia) ${ }^{3}$. La toxicidad hepática, aunque consta en la información del producto, resulta menos conocida, publicándose los dos primeros casos de hepatitis tóxica en 1982. Según la revisión de Bruguera ${ }^{4}$, habían publi- cado 23 casos de hepatitis por ticlopidina hasta 1997 . Se presenta un nuevo caso de hepatitis colestásica probablemente inducida por ticlopidina en mujer anciana hipotiroidea.

Mujer de 81 años con antecedentes de hipotiroidismo primario (en tratamiento sustitutivo con levotiroxina) y artritis microcristalina que presentó un accidente cerebrovascular isquémico hemisférico derecho instaurándose tratamiento con ticlopidina (250 mg) 1 comprimido cada 12 horas y nimodipino 1 comprimido cada 8 horas. Tres semanas después de iniciado el tratamiento, consultó por astenia, prurito, coluria e ictericia. A la exploración física presentaba una tensión arterial de 160/70, rasgos hipotiroideos, e ictericia de piel y mucosas, sin visceromegalias a la palpación abdominal. Los exámenes analíticos mostraban: bilirrubina total, $15 \mathrm{mg} / \mathrm{dl}$; bilirrubina esterificada, $13 \mathrm{mg} / \mathrm{dl}$; colesterol $336 \mathrm{mg} / \mathrm{dl}$; triglicéridos $280 \mathrm{mg} / \mathrm{dl}$; ALT, 419 U/1; AST, 298 U/1, fosfatasa alcalina 1.239 U/1; gamma-GT 904 U/1, y ferritina $1.869 \mathrm{mg} / \mathrm{dl}$. Las hormonas tiroideas fueron normales. Las determinaciones de $\mathrm{HBs} \mathrm{Ag}$, antiVHA-IgM, anti HBc-IgM, anti VHC, anti-CMV IgM, anti-VEB-
IgM, ANA, AMA, AML, y anti LKM fueron negativos. La ecografía y la tomografía computerizada abdominal fueron normales. Se retiró la ticlopidina y nimodipino a su ingreso permaneciendo con cifras elevadas en las primeras cuatro semanas. Las transaminasas y la bilirrubina se normalizaron a los 3 meses y la fosfatasa alcalina al año de seguimiento.

En el caso descrito se observa una estrecha relación entre el inicio de la toma de ticlopidina y el desarrollo de hepatitis. Aplicando los criterios de causalidad sobre los trastornos hepáticos provocados por fármacos ${ }^{5}$, se puede afirmar que la lesión hepática descrita es aguda, de predominio colestásico (ALT/fosfatasa alcalina<2) y muy probablemente inducida por ticlopidina. Podemos sugerir esta etiología tras descartar otras causas de lesión hepática (vírica, infecciosa, inmunológica, alcohólica o metabólica) y por criterior cronológicos: la reacción se manifestó entre los 590 días desde el comienzo de la administración de la ticlopidina y las alteraciones bioquímicas hepáticas descendieron más del $50 \%$ antes de los 6 meses. Ni las enfermedades previas de la enferma ni el resto de fármacos que tomaban jus- 
tifican el cuadro (tiroxina y nimodipino). Sin embargo, no se descarta que alguno de los fármacos o el propio hipotiroidismo influyeran en el daño hepático inducido probablemente por ticlopidina. De acuerdo a los criterios de causalidad de los trastornos hepáticos ${ }^{5}$, no se efectuó una prueba de reintroducción de la ticlopidina.

La toxicidad hepática por ticlopidina resulta menos conocida que otros efectos adversos. En el estudio CATS aparece "alteración de la función hepática" en un $4,4 \%{ }^{4}$. Hay diversas comunicaciones de toxicidad hepática por ticlopidina en literatura nacional ${ }^{4,6-8}$ e internacional $^{1}$. En nuestro entorno, la incidencia notificada de reacciones adversas hepáticas por ticlopidina es de aproximadamente 2 casos por 10.000 tratados $^{2}$, probablemente más bajo de lo esperado por el sesgo de la infranotificación.

La toxicidad hepática inducida por ticlopidina comunicada va desde un aumento de las enzimas hepáticas hasta un cuadro de colestasis aguda ${ }^{2}$. En los casos publicados generalmente la toxicidad hepática cursa como una hepatitis colestásica ictérica (elevación de la bilirrubina y de las enzimas de colestasis más que de las transaminasas) $)^{3,6,8}$; en otros se presenta como hepatitis con colestasis analítica sin ictericia ${ }^{3,6}$, y algunos debutan como colestasis con normalidad de las transaminasas ${ }^{7}$. La disfunción hepática es reversible y se normalizan las alteraciones hepáticas en el primer año, ${ }^{3,46-8}$.

La lesión hepática asociada al tratamiento con ticlopidina se atribuye a un mecanismo de idiosincrasia y se relaciona con algún metabolito, todavía no caracterizado, del fármaco original ${ }^{4}$. En otros casos se baraja como causa del daño hepático un mecanismo de hipersensibilidad al cursar la disfunción hepática con una erupción maculopapulosa y fiebre ${ }^{4}$.

Como Carvajal García-Pando et $\mathrm{al}^{2}$ creemos importante las reacciones adversas hepáticas por ticlopidina y probablemente la relevancia sea mayor de la información publicada hasta la fecha. Por ello,
Torrano Larrión ${ }^{6}$ considera que se debería monitorizar en los que ini cian tratamiento con ticlopidina las pruebas de función hepática en los primeros tres meses.

\section{Ortiz Marín, J. M. Ramos Rincón*，F. Alberca**，J. P. Arribas Ros*}

MIR de Medicina Familiar y Comunitaria. *Servicio de Medici na Interna. **Sección de Aparato Digestivo. Hospital “J.M. Morales Meseguer”. Murcia.

\section{BIBLIOGRAFÍA}

1. Cassidy LJ, Schuster BG, Halparin LS Probable ticlopidine-induced cholestatic hepatitis. Ann Pharmacother 1995; 29; 30-2.

2. Carvajal García-Pando A, García Orte ga P, Rueda de Castro AM, García del Pozo J. Ticlopidina y reacciones adversas hepáticas. Datos recogidos por el Sistema Español de Farmacovigilancia. Med Clin (Barc) 1999; 112; 557-8.

3. Gent M, Easton JD, Hachinski VC, Panak E, Sicurella J, Blakey JA, et al. The Canadian American ticlopidine study (CATS) in tromboembolic stroke. Lancet 1989, 1: 1215-20.

4. Brugüera M. Hepatitis colestásica asociada a ticlopidina. Gastroenterol Hepatol 1997, 20: 163-4.

5. Benichou C. Criteria of drug-induced liver disorders: reports of an international consensus meeting. J Hepatol 1990 272-6.

6. Torrano Larrión F. Hepatitis colestásica por ticlopidina. Aten Prim 1999; 24 : 115

7. Díaz Fuenzalida A, Valdés Socín $H$, Laudano O, Avagnina A, Findor JA. Colestasis asociada a ticlopidina. Gastroenterol Hepatol 1997; 20: 128-30.

8. Artímez ML, Fernández E, Rodríguez M, González M, Rodrigo L. Hepatitis tóxica por ticlopidina. Tres nuevos casos. Rev Esp Enf Digest 1997; 89: 796-7.

\section{Tiroiditis postparto: a} propósito de dos casos

\section{Sr. Director:}

La tiroiditis postparto es una variación de la tiroiditis silente, como en ella se produce una inflamación del tiroides de probable etiología autoinmune y con una evolución subaguda ${ }^{1}$. Generalmente aparece entre los 2 y los 5 meses tras el parto ${ }^{2}$.

$\mathrm{Su}$ incidencia oscila entre el 5$6 \%$ de la población general ${ }^{2}$. Histológicamente se produce una infiltración linfocitaria de la glándula que se traduce en una fase de hiperfunción seguida de otra de hipofunción. La mayoría evolucionan hacia la recuperación completa, pero hasta en un $25 \%$ de los casos el hipotiroidismo es permanente $^{2,3}$.

Es característica la elevación moderada de los anticuerpos antiperoxidasa tiroidea. Estos hallazgos unidos al momento característico de aparición de la enfermedad permiten llegar al diagnóstico, aunque para realizar un diagnóstico de certeza es necesario el estudio histológico.

\section{PRESENTACIÓN DE LOS CASOS}

\section{Caso 1}

Mujer de 35 años que consulta por un cuadro de astenia, mialgias, dificultad de concentración y aumento de $3 \mathrm{~kg}$ de peso desde el parto 5 meses antes, la lactancia es materna. La exploración física es anodina. Se realiza una analítica de rutina en la que se encuentra: colesterol=286 mg/dl (150-200 $\mathrm{mg} / \mathrm{dl}), \mathrm{GOT}=58 \mathrm{UI} / \mathrm{l}$ (5-45 UI/l) , $\mathrm{GPT}=52 \quad \mathrm{UI} / 1 \quad(5-45 \quad \mathrm{UI} / \mathrm{l}) \quad \mathrm{y}$ $\mathrm{LDH}=573 \mathrm{UI} / 1$ (90-230 UI/l). Ante estas alteraciones se amplía el estudio analítico encontrándose unas hormonas tiroideas alteradas: $\mathrm{TSH}=67,1 \quad(0,4-3,1), \quad \mathrm{T} 4=0,09$ $(0,78-2,01)$.

Estos resultados muestran un hipotiroidismo; ante la sospecha de que la causa sea una tiroiditis postparto se solicitan anticuerpos antitiroideos: antiperoxidasa= $14.454 \mathrm{UI} / \mathrm{ml}(<12 \mathrm{UI} / \mathrm{ml})$ y antitiroglobulina $=249,8 \mathrm{UI} / \mathrm{ml} \quad(<60$ $\mathrm{UI} / \mathrm{ml}$ ). Estos resultados apoyan el diagnóstico. Se inicia el tratamiento del hipotiroidismo con hormona tiroidea, lo que hace remitir la sintomatología. Después de tres 
meses se suspende el tratamiento para ver si ha habido recuperación de la glándula, pero a las tres semanas reaparece la sintomatología y se vuelven a alterar las hormonas: $\mathrm{TSH}=72,6$ y T4=0,19. Se repite la operación a los tres meses con idéntico resultado, por lo que se concluye que la lesión glandular es irreversible y el hipotiroidismo permanente.

\section{Caso 2}

Paciente de 32 años que consulta por dolor cervical y odinofagia. Entre sus antecedentes personales sólo destaca un parto eutócico 3 meses antes. En la exploración física se aprecia un tiroides aumentado de tamaño y ligeramente doloroso a la palpación. Se solicita una analítica con hormonas tiroideas en la que: $\mathrm{TSH}=0,01$ y $\mathrm{T} 4=2,95$, anticuerpos antiperoxidasa $=179,5 \mathrm{y}$ antitiroglobulina=63.

En la ecografía se encuentra una glándula aumentada de tamaño y de ecogenicidad difusamente alterada sin lesiones ocupantes de espacio que no permite diferenciar entre bocio multinodular y tiroiditis evolucionada. Todos estos datos nos llevan al diagnóstico de una tiroiditis postparto. Dado que la alteración hormonal no es importante no se instaura tratamiento, se mantiene el control con analíticas periódicas que muestran una evolución hacia la normofunción tiroidea que permanece estable, lo que confirma el diagnóstico de tiroiditis postparto con recuperación de la función glandular.

\section{DISCUSIÓN}

La tiroiditis postparto es una tiroiditis subaguda que forma parte de las tiroiditis silentes o tiroiditis linfocitarias subagudas, y es de etiología autoinmune ${ }^{1}$. Se presenta con una incidencia que oscila entre el 5-6\% en mujeres sanas hasta un $25 \%$ en pacientes con diabetes tipo $\mathrm{I}^{2}$. También hay estudios que demuestran mayor riesgo en pacientes con enfermedad de Graves previa aunque ésta esté en remisión; de hecho se considera que ésta podría ser la causa de las reagudizaciones de dicha enfermedad que se producen tras el parto ${ }^{4}$.

Histológicamente se produce una infiltración linfocitaria de la glándula que en un primer momento se traduce en una situación de hiperfunción porque se estimula la liberación de la hormona tiroidea almacenada en los folículos. Más adelante se agotan estas reservas y el tejido glandular lesionado no es capaz de sintetizar hormona por lo que se pasa por una fase de hipotiroidismo hasta que se regenere la glándula ${ }^{5}$. Esto sucede en un 75$80 \%{ }^{2}$ de los casos y la recuperación de la función tiroidea es completa, pero hasta en un $25 \%$ de las pacientes, como sucede en nuestro caso 1, la lesión glandular es irreversible y la paciente tendrá un hipotiroidismo permanente ${ }^{2,3}$.

En un $8 \%$ de los casos se presenta de forma asintomática ${ }^{2}$ y en la mayoría la clínica es muy variable. Es frecuente que como en nuestro caso 1, la fase de hipertiroidismo pase desapercibida y el diagnóstico se realice cuando aparecen los síntomas de hipotiroidismo. El caso 2 debuta con un bocio ligeramente doloroso, es típico de esta entidad un bocio pequeño que se encuentra hasta en un $50 \%$ de los casos ${ }^{2}$, pero a diferencia de nuestra paciente es indoloro y homogéneo.

Aunque el diagnóstico de certeza sólo es posible mediante el exámen histológico, se puede llegar a un alto índice de sospecha de forma indirecta. Es típica la elevación de los anticuerpos antiperoxidasa a título no muy elevado ${ }^{6}$. Además en las mujeres que presentan títulos elevados de estos anticuerpos durante el embarazo se produce la tiroiditis entre un 35 y un $85 \%$ según las series ${ }^{2}$. Así la elevación de estos anticuerpos junto con el momento de presentación del cuadro y su evolución permiten hacer una adecuada aproximación diagnóstica, como sucede en nuestros casos 1 y 2 .

La aparición de una tiroiditis postparto predice la aparición de un nuevo brote en sucesivos embarazos. Por ello es necesario que el médico de familia haga un correcto y oportuno diagnóstico; además por la forma de presentación de la enfermedad anteriormente descrita, es el médico de Atención Primaria quien es testigo de las diferentes fases y si lo sospecha puede hacer el diagnóstico y tratamiento con los medios técnicos que tiene al alcance.

\section{D. Cano Pérez, M. I. Contre- ras Calzada, M. D. Conejos Miquel, M. B. Martínez Urroz}

\section{Médicos de Familia. \\ Centro de Salud "La Chopera I". Alcobendas. Madrid.}

\section{BIBLIOGRAFÍA}

1. Díez JJ. Tiroiditis silente y tiroiditis posparto. Aten Prim 1995; 16: 147-50.

2. Girón JA, Guerrero F, Telléz F. Tiroiditis. Medicine 2000; 8: 895-902.

3. Learoyd DL, Fung HYM, McGregor AM. Postpartum thyroid dysfunction. Thyroid 1992; 2:73-80.

4. Mestman JH, Goodwin TM, Montoro MM. Thyroid disorders of pregnancy. Endocrinol Metab Clin North Am 1995; 24 : 41-71.

5. Mizukami Y, Mighigishi T, Nonomura A, Hashimoto T, Nakamura S, Tonami N, et al. Postpartum Thyroiditis. A clinical, histologic, and immunopathologic study of 15 cases. Am J Clin Pathol 1993; 100: 200-5.

6. Gerstein H. How common is postpartum thyroiditis? A methodologic overview of the literature. Arch Intern Med 1990; 150: 1397-400. 